Background Maintaining healthy ageing (HA) is a crucial priority in older adults worldwide, given global population ageing, increased number of years living with disability, and the need for new treatments. Omega-3 polyunsaturated fatty acids (n3-PUFA) from seafood and plants exert favourable physiologic effects that could benefit HA. However, relationships between n3-PUFA and HA are not well-established, especially using serial biomarkers which provide highly objective measures.

Methods We investigated the longitudinal association between serial circulating $\mathrm{n} 3$-PUFAs and maintenance of $\mathrm{HA}$ in the Cardiovascular Health Study, evaluating 2342 older U.S. adults with mean age 75 years and successful HA to-date at baseline in 1992-93. Individual plasma phospholipid n3-PUFAs (expressed as $\%$ of total fatty acids) including alpha-linoleic acid (ALA), eicosapentaenoic acid (EPA), docosapentaenoic acid (DPA), and docosahexaenoic acid (DHA) were quantified using gas chromatography in 1992-93, 1998-99, and 200506. HA was defined as survival free of cardiovascular disease, cancer, lung disease, and severe chronic kidney disease, with no difficulties with activities of daily living and intact cognitive function (Mini-Mental State Examination $\geq 80^{\text {th }}$ percentile); dying with a lifetime meeting this criteria was also considered as HA. Events were centrally adjudicated or determined from medical records and diagnostic tests. Multivariable-Cox proportional hazards models with time-varying covariates evaluated the association between time-varying, cumulative average n3-PUFAs and unsuccessful HA.

Results During 22 years of follow-up, 267 (11\%) participants experienced successful HA. After multivariable-adjustments, the interquintile range of total n3-PUFAs and seafood-derived n3PUFAs was associated with lower risk of unsuccessful HA by $17 \%(0.74 \%-0.93$ 95\% CI, $\mathrm{p}=0.002)$ and 16\% (0.75\%-0.94 $95 \% \mathrm{CI}, \mathrm{p}=0.002)$, respectively. Individually, EPA, DPA and DHA each associated with lower risk of unsuccessful HA by $12 \% \quad(0.80 \%-0.97 \quad 95 \% \mathrm{CI}, \quad \mathrm{p}=0.009), \quad 14 \% \quad(0.77 \%-0.97$ $95 \% \mathrm{CI}, \mathrm{p}=0.010)$ and $15 \%(0.76 \%-0.9695 \% \mathrm{CI}, \mathrm{p}=0.009)$, respectively. Plant-derived ALA levels were not significantly associated with HA. Sensitivity analyses including freedom from atrial fibrillation, milder chronic kidney disease, and diabetes within the HA definition did not appreciably alter results.

Conclusion Among older adults with mean age 75 years and HA to-date, a higher cumulative level of circulating seafoodderived n3-PUFAs (combined and individually), but not plantderived ALA, was associated with maintainence of HA. These novel findings support guidelines for increased fish intake among older adults; and need for further investigations into plausible biological mechanisms and interventions for effects of n3-PUFAs on maintenance of HA.

\section{OP70 WEALTH DIFFERENCES IN AGE-TRAJECTORIES OF BODY SIZE: FINDINGS FROM THE ENGLISH LONGITUDINAL STUDY OF AGEING}

${ }^{1} \mathrm{C}$ Lassale ${ }^{*},{ }^{1} \mathrm{~A}$ Steptoe, ${ }^{2} \mathrm{P}$ Zaninotto. ${ }^{1}$ Behavioural Science and Health, University College London, London, UK; ${ }^{2}$ Epidemiology and Public Health, University College London, London, UK

\subsection{6/jech-2017-SSMAbstracts.69}

Background While the obesity epidemic has devastating health consequences at all ages, underweight is also associated with an increased mortality risk. Lower socioeconomic status is associated with higher obesity rates and greater weight gain, but evidence from prospective studies in older adults is scarce. Our aim was to describe age-trajectories of body mass index (BMI) and waist circumference (WC) in a population-based study of older adults in England and to assess the association with wealth.

Methods Data come from a nationally representative sample of 3259 men and 3966 women aged 52y and over from the English Longitudinal Study of Ageing (ELSA) who had a measurement of BMI and WC on three occasions (2004-2005; 2008-2009; 2012-2013). We used latent growth curve modelling to estimate baseline status (intercept) and rate of change (linear slope) interpreted as the change per year. Intercept and slope were regressed on wealth tertile, and covariates (age, ethnicity, marital status, physical inactivity, smoking status and limiting long-standing illness). Gender- and age-specific $(<70, \geq 70 \mathrm{y})$ models were fitted.

Results In the $<70$ y group, a man aged $60 \mathrm{y}$ in the richest wealth tertile had a baseline BMI of $27.7 \mathrm{~kg} / \mathrm{m}^{2}$ and WC of $100.5 \mathrm{~cm}$ and a woman a BMI of $26.9 \mathrm{~kg} / \mathrm{m}^{2}$ and WC of $88.2 \mathrm{~cm}$. BMI increased by $0.04 \mathrm{~kg} / \mathrm{m}^{2}$ every year in men and $0.05 \mathrm{~kg} / \mathrm{m}^{2}$ in women; and WC increased by $0.15 \mathrm{~cm} /$ year in men and $0.21 \mathrm{~cm} /$ year in women. Being in the poorest wealth group was associated with highest baseline BMI $\left(28.7 \mathrm{~kg} / \mathrm{m}^{2}\right.$ for men and $29.4 \mathrm{~kg} / \mathrm{m}^{2}$ for women) and WC $(103.1 \mathrm{~cm}$ in men and $93.6 \mathrm{~cm}$ in women). However, there was no difference in the rate of change between those in the richest and poorest wealth tertiles. In the $\geq 70 \mathrm{y}$ group, for a man aged $77 \mathrm{y}$ in the richest tertile, the baseline BMI was $26.7 \mathrm{~kg} / \mathrm{m}^{2}$ and WC $100.0 \mathrm{~cm}$ and for a woman it was $26.3 \mathrm{~kg} / \mathrm{m}^{2}$ and $88.7 \mathrm{~cm}$. The rate of change was non-significant for both anthropometrics markers. An individual of the same age in the poorest tertile had higher baseline BMI and WC (man: $27.5 \mathrm{~kg} / \mathrm{m}^{2}$ and $102.1 \mathrm{~cm}$; woman: $28.1 \mathrm{~kg} / \mathrm{m}^{2}$ and $92.1 \mathrm{~cm}$ ).

Conclusion In this population-based study, BMI and WC increased significantly over time in both men and women until the age of 70 , thereafter it remained stable. Less wealth was strongly associated with a higher BMI and WC at any given age, however the rates of change were similar, indicating that the socioeconomic gap associated with excess adiposity did not close with ageing.

\section{OP71 THE EFFECT OF LONGITUDINAL CHANGES IN PHYSICAL AND MENTAL HEALTH ON CONTINUING SOCIAL PARTICIPATION IN OLDER IRISH ADULTS: ANALYSIS FROM THE IRISH LONGITUDINAL STUDY OF AGEING}

${ }^{1} \mathrm{~S}$ Leahy, ${ }^{1} \mathrm{C}$ McGarrigle*, ${ }^{1} \mathrm{D}$ Carey, ${ }^{1,2} \mathrm{RA}$ Kenny. ${ }^{1}$ The Irish Longitudinal Study on Ageing, Trinity College Dublin, Dublin, Ireland; ${ }^{2}$ Mercer's Institute for Successful Ageing, St. James's Hospital, Dublin, Ireland

\subsection{6/jech-2017-SSMAbstracts.70}

Background Social engagement and participation in leisure activities are recognised as beneficial to the physical and mental health and wellbeing of older adults and have been shown to lower the risk of negative health outcomes and early mortality. Identifying factors that constrain or enable social participation in older age can help to facilitate continuing engagement and thus improve future health outcomes. This study aimed to investigate the longitudinal relationship between accrual of chronic health conditions and changes in depressive symptoms and continuing social participation in community dwelling older adults over a 4 year period. 